\title{
RESIDUAL GENERATORS FOR DAE SYSTEMS UTILIZING MINIMAL SUBSETS OF MODEL EQUATIONS
}

\author{
Jonas Biteus*, Mattias Nyberg** \\ * Division of Vehicular Systems, Dept. of Electrical Engineering \\ Linköpings universitet, SE-58183 Linköping, Sweden \\ E-mail: biteuscisy. Iiu.se \\ ** Software and Diagnostics, Engine Development \\ SCANIA AB, SE-151 87 Södertälje, Sweden \\ E-mail: mattias.nyberg@scania.com
}

\begin{abstract}
A common approach to design diagnostic systems is to use residual generators. These generators are usually constructed considering all the model equations. However, there are several advantages of instead consider small subsets of model equations, so called minimal structurally singular (MSS) sets of equations. This paper presents a new method for finding residual generators for MSS sets. A special property of the MSS set, namely that it is minimally over determined, is utilized. Two approaches are considered, one which is based on the use of a dynamic numerical equation solver, and another which uses a static numerical equation solver. The approaches are demonstrated on a non-linear point-mass satellite system. Copyright (C)2002 IFAC.
\end{abstract}

Keywords: Fault diagnosis; Residual generator; Fault detection; Fault isolation; Diagnosis; Structural analysis; Differential equations.

\section{INTRODUCTION}

When designing model-based fault-diagnosis systems, residual generators are often used. Different subsets of residual generators are sensitive to different subsets of faults, and thereby isolation can be achieved. Each residual does not measure the validity of all model equations in the model, but instead a smaller subset of equations. Given a set of model equations, a first step towards finding residual generators, is to find all minimal structurally singular (MSS) sets of equations (also called e.g., minimal over-determined equationsets).

The reason for using MSS sets is that these sets are the smallest sets of equations that can be used to form residual generators. The MSS property is further described in Section 2.

This paper presents a new method for constructing residual generators for MSS sets. A special property for an MSS set is that there is exactly one more equation than unknown variables, this property is utilized by using the extra equation, to define the residual component. The MSS set with a residual component is a residual generator. A more general framework will be presented in Section 3.

Compared to the general residual generation problem, where the whole model is usually considered, there are two advantages to start with an MSS set. The first is that this set is typically small, which follows from the fact that it is minimal. A small model is normally easier to handle than a large model. The second advantage is that decoupling of faults and disturbances has already been achieved, i.e., further decoupling is not necessary.

When extracting the set of MSS sets, different assumptions about the structural relationship between the unknown variables and about derivatives of sensor values, will result in different sets of MSS sets. From these assumptions two main approaches, the dynamic and the static approach, are found. The dynamic approach results in a set of equations that includes $d y$ namic state variables, while the static approach results in a set of equations with only instantaneous state variables (also called e.g., algebraic state variables). It is also possible to make a combination of the two approaches, this will be called the partially dynamic approach. These different approaches are discussed in deeper detail in Section 4.

There are constraints on how the residual component is defined. Due to structurally and analytically properties for the MSS set, only the equations that are analytically redundant can be used to define the residual component, this is discussed in Section 5. Further, the residual component must be added in such a way, that the residual generator is stable. This will be briefly discussed in Section 6.

An example of a satellite system is analyzed in Section 7, and finally, Section 8 gives the conclusions. 


\section{MSS SETS OF EQUATIONS}

The main property of an MSS set is that there is exactly one more equation than unknown variables included in the equations, i.e., it is structurally singular. Another important property is that no proper subset of the MSS set is an MSS set, i.e., it is minimal.

In (Krysander and Nyberg, 2002) a systematic and automatic algorithm is derived, called the MSS-algorithm. The algorithm finds the simplest set of MSS sets with the highest possible diagnosis capability. This algorithm will be used in this paper.

The input to the algorithm is a structural model and information about which derivatives of sensor values that can be approximated. The structural model includes information about the connection between unknown variables and equations. By analyzing and manipulating this structural model, the algorithm finds all MSS sets and then selects the set of MSS sets with highest possible diagnosis capability.

The output of the algorithm is a set of MSS sets where each MSS set might include differentiated and nondifferentiated equations from the original model.

\section{PROBLEM FORMULATION}

The problem studied in this work can be described as follows. Given a set of MSS sets, $\left\{\mathrm{MSS}^{1}, \ldots, \mathrm{MSS}^{k}\right\}$, input data $u=\left\{u_{u}, u_{y}\right\} \in U$, where $u_{u}$ is a vector of control signals and $u_{y}$ is a vector of sensor values, find a residual generator for each MSS set.

Assume that MSS set $i$, denoted $M S S^{i}$, is a differential algebraic system of the form

$$
0=G^{i}\left(\dot{x}^{i}, x^{i}, z^{i}, u^{i}, f^{i}\right)
$$

where $x^{i} \in X$ is a vector of dynamic state variables, $z^{i} \in Z$ is a vector of instantaneous state variables, and $f^{i}$ is a vector of unknown faults which are assumed to be zero in the fault free case. The $\operatorname{dim}\left(G^{i}\right)=$ $\operatorname{dim}\left(\dot{x}^{i}, x^{i}, z^{i}\right)+1$, where $\operatorname{dim}(\cdot)$ is the dimension, and $\dot{x}^{i}, x^{i}, z^{i}$ are the unknown variables. It is further assumed that some initial values satisfying (1) have been found. Superscript index will be used to denote the corresponding MSS set, while subscript will be used for vector and matrix components. The indexes will be dropped when there is no risk of confusion.

The state variable and input data space is

$$
\Omega=X \times Z \times U .
$$

The space $\Omega$ includes knowledge of the system that can be used when constructing the residual generator. For physical systems, $\Omega$ will often include physical limitations for the state variables.

The main idea is to add a scalar residual component, $r \in \mathbb{R}^{1}$, to the MSS set and use a numeric solver to find an approximation of the value of the residual component. Let $n$ denote the highest derivative of $r$.

If a dynamic solver is used, the highest derivative of the residual component, $r^{(n)}$, is unknown, while the lower derivatives are known from integration of $r^{(n)}$.
The adding of this unknown variable to an MSS set gives a system of equations with as many equations as unknown variables. The resulting set of equations will be called an MSS model (slight misuse of the acronym MSS). If this MSS model is solvable and stable it will be used as a residual generator.

How the residual component and its derivatives are added is a freedom that can be used when designing the MSS model. With a parameter matrix $\Gamma \in$ $\mathbb{R}^{\operatorname{dim}(G) \times n+1}$, the MSS model is

$$
0=G(\dot{x}, x, z, u)+\Gamma R
$$

where $R=\left[r, \dot{r}, \ldots, r^{(n)}\right]^{T}$. There are some constraints on the values of $\Gamma$, and these will be discussed later in Section 5 and 6.

An example: Assume that a model is described by

$$
\begin{array}{ll}
e_{1}: \dot{\rho}=u_{1}+f+\sigma & e_{2}: \sigma+u_{2}=0 \\
e_{3}: y_{1}=\rho+\sigma & e_{4}: y_{2}=\rho
\end{array}
$$

An MSS set is $\left\{e_{1}, e_{2}, e_{4}\right\}$, and an MSS model, i.e., Eq. (3), for this set with $n=1$ is

$$
0=\left[\begin{array}{c}
\dot{\rho}-u_{1}-\sigma \\
\sigma+u_{2} \\
y_{2}-\rho
\end{array}\right]+\Gamma\left[\begin{array}{l}
r \\
\dot{r}
\end{array}\right]
$$

where $\Gamma \in \mathbb{R}^{3 \times 2}$. Simulations of this MSS model will give a residual $r$ that is sensitive to fault $f$, if fault $f$ is sufficiently large.

\section{APPROACHES TO EXTRACT MSS SETS}

When the MSS sets are extracted, different assumptions about the structure of the system, will result in different sets of MSS sets. These different assumptions give the dynamic, static and partially dynamic approaches. These three approaches will be discussed in this section.

\subsection{Dynamic Approach}

The dynamic approach arises from the assumption that a dynamic solver is available. Further it is assumed that no derivatives of sensor values can be approximated, e.g., sensor value $u_{y_{1}}$ is known while its derivative $\dot{u}_{y_{1}}$ is unknown. When a dynamic solver is used, the state variable is known from the integration of its derivative. Therefore, $\dot{x}$ is assumed unknown while $x$ is known.

The MSS-algorithm is given the structural model, where $\dot{x}$ and $z$ are unknown. The output is a set of MSS sets. The sets might include differentiated and non-differentiated equations. Further, the MSS model might be a non-linear DAE, therefore, a non-linear DAE solver is preferred.

The benefit with the dynamic approach is that derivatives of sensor values does not have to be approximated. The disadvantage is solution stability and complexity due to models with index higher than zero.

To continue the example above. The MSS set $\left\{e_{1}, e_{2}, e_{4}\right\}$ is found with the dynamic approach. In this set, $x=$ $\{\rho\}, z=\{\sigma\}$ and $u=\left\{u_{1}, u_{2}, y_{2}\right\}$. 


\subsection{Static Approach}

The static approach arises from the assumption that a dynamic solver is not available. Because of this, many equations that includes derivatives of state variables are unusable. This will for most physical problems severely restrict the number of MSS sets and thereby the diagnosability. To increase the diagnosability, assume that derivatives of sensor values up to some given order can be approximated.

Since no dynamic solver is available in this approach, the structural relationship between a state variable and its derivative does not exist. To reduce the risk of confusion let $x_{D} \triangleq \dot{x}$. Further, no derivatives of $r$ are included, i.e., $n=0$.

The MSS-algorithm is given the structural model, where $x_{D}, x$ and $z$ are unknown. The output from the algorithm is a static system of equations that can be solved with a general equation solver. The approach can successfully handle a larger class of MSS sets than the consistency relation approach used in (Krysander and Nyberg, 2002).

The benefit with the static approach is simplicity when constructing the residual generator. The disadvantage is that, often, derivatives of sensor values have to be approximated.

To continue the example above. With the static approach the MSS set $\left\{e_{1}, e_{2}, \dot{e}_{4}\right\}$ is found, where equation $\dot{e}_{4}$ is the time derivative of equation $e_{4}$, see Section 2. In this set, $x_{D}=\left\{\rho_{D}\right\}, x=\{\rho\}, z=\{\sigma\}$ and $u=\left\{u_{1}, u_{2}, y_{2}^{D}\right\}$.

\subsection{Partially Dynamic Approach}

The dynamic and static approach are two extremes. It is possible to make a combination of the two approaches. If a subset of derivatives of sensor values can be approximated the MSS models will be dynamic models with some dynamic states removed.

The main benefit is when the MSS models have a high index. By careful selection of which sensor values to approximate, the index can be reduced.

\section{REDUNDANT EQUATIONS}

It is not possible to add $r^{(n)}$ to the MSS set arbitrary. Due to structural and analytical properties for the set, there are constraints that $\Gamma$ must fulfill.

First, $r^{(n)}$ must be added to at least one of the analytically redundant equations. An equation is structurally redundant if, when the equation is removed, the remaining set of equations is exactly solvable for some operating point in $\Omega$. It is analytically redundant if, it is structurally redundant for all operating points in $\Omega$. Note that an equation is structurally redundant if it is analytically redundant.

Secondly, if it is added to several analytically redundant equations, $\Gamma$ must fulfill some additional constraints, discussed later in Section 5.2.
In this section methods to find all structurally and analytically redundant equations will be presented. The structurally redundant equations can be found with structural or analytical methods, while the analytically redundant equations can only be guaranteed to be found with analytical methods.

The main idea when searching for redundant equations is to add $r^{(n)}$ to each equation

$$
0=G(\dot{x}, x, z, u)+\bar{\Gamma} r^{(n)}
$$

where $\bar{\Gamma}$ is the last column in $\Gamma$. If (4) is exactly solvable then at least one of the equations is structurally or analytically redundant.

\subsection{Finding Structurally Redundant Equations}

To find the structurally redundant equations, structural analysis can be used. The structural analysis finds a bipartite matching between equations and unknown variables (Krysander and Nyberg, 2002; Harary, 1969).

If equation $j$ is matched to $r^{(n)}$, it is structurally redundant. To find another structurally redundant equation, let $\bar{\Gamma}_{j}=0$ and find a new bipartite matching. Suitable repetition gives all structurally redundant equations.

The example in Section 3 can be represented by the structural graph shown in the left part of Fig. 1. The first row includes the equations, and the second the unknown variables. The structural relationships are shown with thin arrows. In the right part, a bipartite matching algorithm has been used to find a bipartite matching. The matching show that $e_{4}$ is a structurally redundant equation. Remove the relationship and find a new matching. In this example it is not possible to find any new bipartite matching, and the conclusion is that only equation $e_{4}$ is structurally redundant.
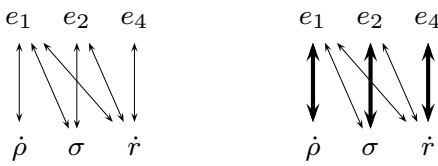

Fig. 1. Structural model (left) and a bipartite matching.

\subsection{Finding Analytically Redundant Equations}

To find the analytically redundant equations the implicit function theorem will be used. To investigate if a system of equations fulfills the theorem, a common approach is to test if the Jacobian is nonsingular (Venkatasubramanian et al., 1995; Mattsson and Söderlind, 1992). Eq. (4) will only fulfill the theorem if $r^{(n)}$ has been added to a structurally redundant equation.

For the dynamic approach this means that

$$
|J|=\left|\frac{\partial\left(G(\dot{x}, x, z, u)+\bar{\Gamma} r^{(n)}\right)}{\partial\left[\dot{x}, z, r^{(n)}\right]^{T}}\right|=\xi^{T}(\dot{x}, x, z, u) \bar{\Gamma} \neq 0
$$

where $\xi(\cdot)$ is a vector. The sum $\xi^{T} \bar{\Gamma}$ arises from the definition of determinant, i.e., sum of vector component times co-factor. 
For the static approach,

$$
|J|=\left|\frac{\partial\left(G\left(x_{D}, x, z, u\right)+\bar{\Gamma} r^{(n)}\right)}{\partial\left[x_{D}, x, z, r^{(n)}\right]^{T}}\right|=\xi^{T}\left(x_{D}, x, z, u\right) \bar{\Gamma} \neq 0 .
$$

Now, let $\bar{\Gamma}=\left[0, \ldots, \bar{\Gamma}_{j}, \ldots, 0\right]^{T}$, where $\bar{\Gamma}_{j} \neq 0$. If $|J| \neq 0$ then equation $j$ is structurally redundant.

To see if equation $j$ is analytically redundant the variable space, $\Omega$ has to be considered. For the dynamic case, if

$$
\nexists(\dot{x}, x, z, u): \xi_{j}(\dot{x}, x, z, u)=0 \wedge(x, z, u) \in \Omega
$$

then equation $j$, is guaranteed to be analytically redundant. The meaning is that, if it is not theoretically possible for $\xi_{j}$ to equal zero, then equation $j$ is analytically redundant. Suitable change of unknown variables give the static case.

This section have stated constraints on $\Gamma$ that must be fulfilled. The conclusion is, if $r^{(n)}$ is added to several equations, then $\Gamma$ must fulfill $\xi(\dot{x}, x, z, u) \bar{\Gamma} \neq 0$ for all $(x, z, u) \in \Omega$. A special case is when $r^{(n)}$ is added to only one equation, then it is necessary that this equation is analytically redundant, i.e., fulfills (5).

\section{STABILITY}

From Section 5 some constraints on $\Gamma$ have been stated. The problem now is to find the additional constraints on $\Gamma$ that guarantees stability and give a good fault sensitivity for the residual, while fulfilling the constraints from Section 5. Depending on if the MSS model is linear, bilinear, non-linear, etc., different methods can be used to find the constraints. The problem is similar to the problems faced in FDI observer theory, see for example (Chen and Patton, 1999).

Note, the highest derivative of $r$ can be chosen to simplify the stability analysis. The stability problem will not be further studied in this paper.

\section{SATELLITE EXAMPLE}

This example is a non-trivial non-linear point-mass satellite system. It is taken from (Rugh, 1996; Persis and Isidori, 2001). First the model is presented, Section 7.1. After this, the dynamic, static and partially dynamic approaches are considered, Section 7.2, 7.3, and 7.4 respectively. Last, some simulation results are presented, Section 7.5.

\subsection{Physical Model}

The equations describing the model are

$$
\begin{array}{ll}
e_{1}: \dot{\rho}=v & e_{2}: \dot{v}=\rho \omega^{2}-\theta_{1} \frac{1}{\rho^{2}}+\theta_{2} u_{1}+d \\
e_{3}: \dot{\varphi}=\omega & e_{4}: \dot{\omega}=-\frac{2 v \omega}{\rho}+\theta_{2}\left(\frac{u_{2}}{\rho}+\frac{f_{u_{2}}}{\rho}\right) \\
e_{5}: 0=-y_{1}+\rho & e_{6}: 0=-y_{2}+\varphi+f_{\varphi} \\
e_{7}: 0=-y_{3}+\omega, &
\end{array}
$$

where: $\rho, v$ are radius and radius speed; $\varphi, \omega$ are angle and angular speed; $u_{1}$ is radial and $u_{2}$ tangential thrust; $y_{1}, y_{2}$ and $y_{3}$ are sensor signals; $d$ unknown disturbance; $f_{u_{2}}, f_{\varphi}$ bias fault in $u_{2}$ and $y_{2}$ respectively; $\theta_{1}$ and $\theta_{2}$ are known constants.

The variable space is

$$
\begin{aligned}
& X, Z=\{\rho>0, \omega>0, v, \varphi\} \\
& U=\left\{\left|u_{1}\right| \leq 1,\left|u_{2}\right| \leq 1, y_{1}>0, y_{3}>0, y_{2}\right\} \\
& F=\left\{\left|f_{u_{2}}\right| \leq 1, f_{\varphi} \geq-\varphi\right\} .
\end{aligned}
$$

The variable space limits the values for the state variables to positive radius and angular speed, etc.

\subsection{Dynamic Approach}

The MSS-algorithm is given the structural model and the information that no derivatives of sensor values can be approximated, see Section 4.1. The result is ten different MSS sets and here four of the MSS sets are chosen. These sets are chosen because they have the desired detection and isolation property and are least complex (with respect to number of included equations).

The set of MSS sets is

\begin{tabular}{l|l|ccc} 
MSS & Equation set & $f_{u_{2}}$ & $f_{\varphi}$ & $d$ \\
\hline $\mathrm{MSS}^{1}$ & $\left\{e_{3}, e_{6}, e_{7}\right\}$ & 0 & $X$ & 0 \\
$\mathrm{MSS}^{2}$ & $\left\{e_{1}, e_{2}, e_{4}, e_{5}\right\}$ & $X$ & 0 & $X$ \\
$\mathrm{MSS}^{3}$ & $\left\{e_{1}, e_{4}, e_{5}, e_{7}\right\}$ & $X$ & 0 & 0 \\
$\mathrm{MSS}^{4}$ & $\left\{e_{1}, e_{3}, e_{4}, e_{5}, e_{6}\right\}$ & $X$ & $X$ & 0
\end{tabular}

For example, the $\operatorname{MSS}^{1}$ set is $\left\{\dot{\varphi}=\omega, 0=-y_{2}+\right.$ $\left.\varphi, 0=-y_{3}+\omega\right\}$.

An $X$ in position $i, j$ in the incidence matrix, the rightmost part of the table, means that $\mathrm{MSS}^{i}$ might be sensitive to fault $j$. From the incidence matrix it can be concluded that it is theoretically possible to detect and isolate both faults. $\mathrm{MSS}^{2}$ is sensitive for the disturbance, $d$, but has an interesting property, discussed later in Section 7.2.1.

7.2.1. Analytically Redundant Equations The dynamic state variables $\dot{x}$ are for $\mathrm{MSS}^{1}$ and $\mathrm{MSS}^{3}$ model: $\dot{x}^{1}=\{\dot{\varphi}\} ; \dot{x}^{3}=\{\dot{\rho}, \dot{\omega}\}$. Instantaneous state variables $z$ are: $z^{1}=\{\omega\} ; z^{3}=\{v\}$.

The Jacobians for MSS ${ }^{1}$ and MSS $^{3}$ model are

$$
J^{1}=\left[\begin{array}{cc|c}
-1 & 1 & \\
0 & 0 & \bar{\Gamma}^{1} \\
0 & 1 &
\end{array}\right] \quad J^{3}=\left[\begin{array}{ccc|c}
-1 & 0 & 1 & \\
0 & -1 & -\lambda & \bar{\Gamma}^{3} \\
0 & 0 & 0 & \\
0 & 0 & 0 &
\end{array}\right]
$$

where $\lambda=2 \omega / \rho$. The determinant of the Jacobians are $\left|J^{1}\right|=\bar{\Gamma}_{2}^{1}$ and $\left|J^{3}\right|=0$.

From this it is concluded that, in $\mathrm{MSS}^{1}$ model, equation $2\left(e_{6}\right)$ is analytically redundant, and that $\mathrm{MSS}^{3}$ model includes no redundant equations. A similar analysis give that in $\mathrm{MSS}^{2}$ model, equation 4 is analytically redundant, and that $\mathrm{MSS}^{4}$ model includes no redundant equations.

Note, the $\mathrm{MSS}^{3}$ and $\mathrm{MSS}^{4}$ models have index higher than zero. The reason is that it is not possible to calculate $v$ from equations $e_{1}$ or $e_{4}$ (equation $e_{3}$, $e_{5}, e_{6}$ and $e_{7}$ does not include $v$ ). One solution to 
reduce the index is to use an MSS model that includes equation $e_{2}$, then $v$ can be integrated from $\dot{v}$. One such MSS model is $\mathrm{MSS}^{2}$ model. It includes a redundant equation but is sensitive to the disturbance $d$.

7.2.2. Design of $\Gamma$ for $M S S^{1}$ In section 7.2 .1 it was stated that the second equation is analytically redundant. With a first design choice of $\Gamma_{12}=\Gamma_{31}=$ $\Gamma_{32}=0$ and $n=1$, the model is

$$
0=\left[\begin{array}{l}
-\dot{\varphi}+\omega \\
-y_{2}+\varphi \\
-y_{3}+\omega
\end{array}\right]+\left[\begin{array}{cc}
\Gamma_{11} & 0 \\
\Gamma_{21} & \Gamma_{22} \\
0 & 0
\end{array}\right]\left[\begin{array}{l}
r \\
\dot{r}
\end{array}\right]
$$

In this case the model is linear and therefore, linear analysis will be used to find the constraints for $\Gamma_{11}$, $\Gamma_{21}$ and $\Gamma_{22}$. Transform (6) to the frequency domain

$$
r=\frac{y_{2} s-y_{3}}{s^{2} \Gamma_{22}+s \Gamma_{21}+\Gamma_{11}}
$$

which is stable if $\Gamma_{11}>0, \Gamma_{21}>0$ and $\Gamma_{22}>0$ (Routh's theorem). Note that $\Gamma_{22} \neq 0$ and equation 2 is analytically redundant. With this design choice, the model is stable.

Note, even though linear theory was used to find the constraints on $\Gamma$, the methods presented in this paper is not limited to the linear case. In the general nonlinear case, other methods for stability analysis, such as Lyaponov theory, have to be used.

In Section 7.2, four MSS models have been analyzed. Only two of the MSS models includes analytically redundant equations. Constraints on $\Gamma$ for $\mathrm{MSS}^{1}$ model have been stated.

\subsection{Static Approach}

In the static approach the derivatives, up to some order, of all sensor values have to be approximated. Here only the first derivative will be used. The MSS-algorithm is given this information and finds three different MSS sets. The set of MSS sets found is

\begin{tabular}{l|l|ccc} 
MSS & Equation set & $f_{u_{2}}$ & $f_{\varphi}$ & $d$ \\
\hline $\operatorname{MSS}^{\tilde{1}} \tilde{1}$ & $\left\{e_{3}, \dot{e}_{6}, e_{7}\right\}$ & 0 & $X$ & 0 \\
$\mathrm{MSS}^{2}$ & $\left\{e_{1}, e_{4}, e_{5}, \dot{e}_{5}, e_{7}, \dot{e}_{7}\right\}$ & $X$ & 0 & 0 \\
$\operatorname{MSS}^{\tilde{3}}$ & $\left\{e_{1}, e_{3}, e_{4}, e_{5}, \dot{e}_{5}, \dot{e}_{6}, \dot{e}_{7}\right\}$ & $X$ & $X$ & 0
\end{tabular}

Time differentiation of an equation, e.g., $\dot{e}_{6}$, is performed analytically. For example, $\operatorname{MSS}^{\tilde{1}}$ set is $\left\{\varphi_{D}=\right.$ $\left.\omega, 0=-y_{2 D}+\varphi_{D}, 0=-y_{3}+\omega\right\}$.

7.3.1. Analytically Redundant Equations In the MSSĩ set, the unknown variables are $\varphi_{D}$ and $\omega$, similar for the other MSS models. The determinants of the Jacobians are for the three MSS models determined by $\xi$,

$$
\begin{aligned}
& \xi^{\tilde{1}}=[1,1,-1]^{T} \\
& \xi^{\tilde{2}}=[-\lambda,-1,1, \alpha,-\lambda,-\beta,-1]^{T} \\
& \xi^{\tilde{3}}=[\lambda, \beta, 1, \alpha, \lambda, \beta, 1]^{T},
\end{aligned}
$$

where $\left.\alpha=\left(2 v \omega-\theta_{2} u_{2}\right)\right) / \rho^{2}, \beta=2 v / \rho$ and $\lambda=$ $2 \omega / \rho$.

For example, for MSS ${ }^{\tilde{3}}$, equations 3 and 7 are analytically redundant. To check if the remaining equations are analytically redundant, Eq. (5) have to be considered. This corresponds to

$$
\begin{aligned}
& \left\{(\rho, \omega, v, \varphi) \mid \xi_{1}^{\tilde{3}}=\xi_{5}^{\tilde{3}}=\frac{2 \omega}{\rho}=0 \wedge(\rho, \omega, v, \varphi) \in \Omega\right\}=\emptyset \\
& \left\{(\rho, \omega, v, \varphi) \mid \xi_{2}^{\tilde{3}}=\xi_{6}^{\tilde{3}}=\frac{2 v}{\rho}=0 \wedge(\rho, \omega, v, \varphi) \in \Omega\right\} \neq \emptyset \\
& \left.\left\{(\rho, \omega, v, \varphi) \mid \xi_{4}^{\tilde{3}}=\frac{2 v \omega-\theta_{2} u_{2}}{\rho^{2}}=0 \wedge(\rho, \omega, v, \varphi) \in \Omega\right\}\right) \neq \emptyset .
\end{aligned}
$$

From this it can be concluded that equation 2, 4, and 6 are not analytically redundant. It can be noted that, they are structurally redundant.

In Section 7.3, three different MSS models have been found. For the third MSS model, the analytically redundant equations have been stated.

\subsection{Partially Dynamic Approach}

In Section 7.2 it is concluded that the $\mathrm{MSS}^{3}$ model and the $\mathrm{MSS}^{4}$ model does not include any redundant equations. The problem is that it is not possible to directly calculate $v$ from the equation set. A solution is to partially assume that it is possible to approximate derivatives.

In this section it is assumed that $\dot{y}_{1}$ can be approximated. The MSS-algorithm finds ten different MSS sets and here the two MSS sets that corresponds to $\mathrm{MSS}^{3}$ and $\mathrm{MSS}^{4}$ in Section 7.2 are chosen. The difference is that differentiated equation $\dot{e}_{5}$ is included.

The set of MSS sets is

\begin{tabular}{l|l|ccc} 
MSS & Equation set & $f_{u_{2}}$ & $f_{\varphi}$ & $d$ \\
\hline MSS $^{\hat{3}}$ & $\left\{e_{1}, e_{4}, e_{5}, \dot{e}_{5}, e_{7}\right\}$ & $X$ & 0 & 0 \\
MSS $^{\hat{4}}$ & $\left\{e_{1}, e_{3}, e_{4}, e_{5}, \dot{e}_{5}, e_{6}\right\}$ & $X$ & $X$ & 0
\end{tabular}

7.4.1. Analytically Redundant Equations For the two MSS models the dynamic state variable $\dot{x}$ is: $\dot{x}^{\hat{3}}=$ $\{\dot{\omega}\} ; \dot{x}^{\hat{4}}=\{\dot{\varphi}, \dot{\omega}\}$. Instantaneous state variable $z$ is: $z^{\hat{3}}=\left\{\rho, \rho_{D}, v\right\} ; z^{\hat{4}}=\left\{\rho, \rho_{D}, v\right\}$.

The determinant of the Jacobians are: $\left|J^{\hat{3}}\right|=\bar{\Gamma}_{5}^{\hat{3}}$; $\left|J^{\hat{4}}\right|=\bar{\Gamma}_{6}^{\hat{4}}$. This means that for MSS ${ }^{\hat{3}}$ model and MSS $\hat{4}$ model equations 5 and 6 are analytically redundant, respectively.

7.4.2. Design of $\Gamma$ for $M S S^{\hat{3}} \quad$ In Section 7.4.1 it was stated that equation $5\left(e_{7}\right)$ is analytically redundant. The model will be designed with $n=0, \Gamma_{11}=\Gamma_{31}=$ $\Gamma_{41}=0$ and $\Gamma_{51}=1$,

$$
0=\left[\begin{array}{c}
-\rho^{D}+v \\
-\dot{\hat{\omega}}-2 v \hat{\omega} / \rho+\theta_{2} u_{2} / \rho \\
-y_{1}+\rho \\
-y_{1}^{D}+\rho^{D} \\
-y_{3}+\hat{\omega}
\end{array}\right]+\left[\begin{array}{c}
0 \\
\Gamma_{21} \\
0 \\
0 \\
1
\end{array}\right] r .
$$

Transform $r$ to the frequency domain

$$
r=y_{3}-\frac{\theta u_{2} / y_{1}+\Gamma_{21} y_{3}}{s+2 y_{1}^{D} / y_{1}+\Gamma_{21}} .
$$

The model is locally stable if $2 y_{1}^{D} / y_{1}+\Gamma_{21}>0$. A design choice is $\Gamma_{21}=-2 y_{1}^{D} / y_{1}+K_{1}$ where $K_{1}>0$. 

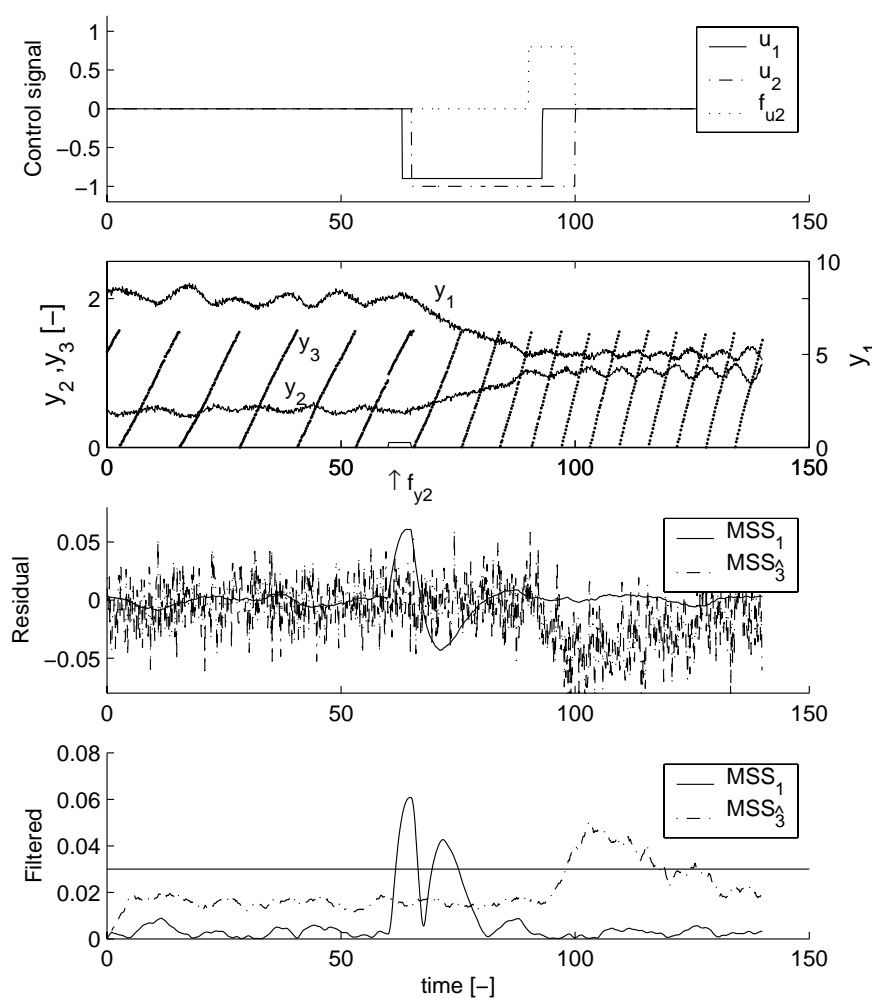

Fig. 2. Residual values.

In Section 7.4 it has been shown that if it is possible to approximate $\dot{y}_{1}$, it is possible to gain simpler MSS models. Two MSS models with analytically redundant equations were found. For MSS ${ }^{\hat{3}}$, constraints on $\Gamma$ has been stated.

\subsection{Simulation}

To test the MSS models analyzed in the example, two MSS models have been implemented. From Section 7.2 is MSS $^{1}$ model chosen and from Section 7.4 is MSS $^{3}$ model chosen. The MSS models are chosen because they are small, simple, and needs the smallest number of approximations of derivatives. $\mathrm{MSS}^{1}$ model is sensitive to fault $f_{\varphi}$ and $\mathrm{MSS}^{\hat{3}}$ model to fault $f_{u_{2}}$. Thereby, full detectability and isolability are achieved.

The chosen MSS models are stable. MSS ${ }^{1}$ model have parameters $\Gamma_{11}=0.2, \Gamma_{21}=2.7$ and $\Gamma_{22}=9 . \operatorname{MSS}^{\hat{3}}$ model have parameter $K_{1}=0.04$.

The physical model and the two MSS models have been implemented in Dymola (Elmqvist, 2002). Fault $f_{\varphi}=15^{\circ}$ is introduced at time $60 \mathrm{~s}$ to $65 \mathrm{~s}$, fault $f_{u_{2}}=0.8$ at time $90 \mathrm{~s}$ to $100 \mathrm{~s}$. Disturbance, $d$, is implemented as white noise. Fig. 2 show the data from the simulation. The upper plot shows control signals and fault $f_{u_{2}}$, second shows sensor values and fault $f_{\varphi}$. Third shows the value of the residuals and the lowest shows the residual components after the use of a moving average filter. Fault $f_{\varphi}$ is detected at time $62 \mathrm{~s}$ and $f_{u_{2}}$ at $98 \mathrm{~s}$. The conclusion is that both MSS models react to correct fault and that detection and isolation are performed correct.
The simulation results show that the two MSS models that have been implemented, react correctly to the introduced faults.

\section{CONCLUSIONS}

Dynamic evaluation of MSS models have been used for fault-diagnosis. These MSS models are residual generators for the original system. An MSS model is formed from a minimal structurally singular (MSS) set of equations with an extra residual component and its derivatives.

Since it is not possible to add the residual component arbitrary, constraints on how the residual component can be added have been stated. The constraints can be divided into two parts, first the component must be added to an analytically redundant equation. Secondly, it must be added in such a way that the MSS model is stable.

The relationship between approximations of derivatives of sensor values and evaluation complexity is studied. It has been shown that it is possible to gain MSS model simplifications at the expense of approximations of derivatives of sensor values. High index MSS models can be relaxed to lower index MSS models if approximations of derivatives are allowed.

The approach has been used on a non-trivial nonlinear point-mass satellite system. MSS models from the dynamic and partially dynamic approach are used and the result is analyzed. Numerical simulation of MSS models shows good results.

\section{REFERENCES}

Chen, J. and Patton, R. (1999), Robust Model-Based Fault Diagnosis for Dynamic Systems, Kluwer Academic Publishers.

Elmqvist, H. (2002), Dymola, User's Manual, 4.2b edn, Dynasim AB, Lund, Sweden.

Harary, F. (1969), Graph Theory, Addison-Wesley Publishing Co., Boston, U.S.A.

Krysander, M. and Nyberg, M. (2002), Structural analysis utilizing MSS sets with application to a paper plant, in '13th International Workshop on Principles of Diagnosis', Semmering, Austria. Dep. report, www.vehicular.isy.liu.se.

Mattsson, S. E. and Söderlind, G. (1992), A new technique for solving high-index DAE using dummy derivatives, in 'Computer-Aided Control System Design', IEEE, Napa, USA, pp. 218-224.

Persis, C. D. and Isidori, A. (2001), 'A geometric approach to nonlinear fault detection and isolation', IEEE Trans. on Automatic Control 46(6), 853865.

Rugh, W. J. (1996), Linear System Theory, 2nd edn, Prentice Hall, Upper Saddle River, USA.

Venkatasubramanian, V. et al. (1995), 'Local bifurcations and feasibility regions in differentialalgebraic systems', IEEE Transactions on Automatic Control AC-40(12), 1992-2013. 